\title{
Monitoring polymorphic transition of a cocrystal to a salt using time-resolved X-ray powder diffraction and solid-state nuclear magnetic resonance spectroscopy
}

\author{
Luzia S. Germann, ${ }^{1}$ Mihails Arhangelskis, ${ }^{3}$ Robin S. Stein, ${ }^{1}$ Robert E. Dinnebier, ${ }^{2}$ Tomislav Friščić ${ }^{1}$ \\ ${ }^{1}$ Department of Chemistry, McGill University, H3A 0B8, Montreal, Canada, ${ }^{2}$ Max-Planck-Institute for Solid State Research, 70569 \\ Stuttgart, Germany, ${ }^{3}$ Faculty of Chemistry, University of Warsaw, Poland. \\ Luzia.germann@mail.mcgill.ca
}

Crystal engineering has emerged as an important field of solid-state chemistry, developing tools to deliberately design functional organic solids. A particularly exciting aspect of crystal engineering is the tuneability of physicochemical properties of organic solids such as solubility, thermal stability, bioavailability etc. without altering the underlying molecular structure(s) - a concept of high relevance for pharmaceutical industry. ${ }^{[1]}$ Altering physisochemical properties can be achieved by relying on different solid forms, such as polymorphs, cocrystals, and salts. ${ }^{[2]}$ The latter two are multicomponent systems that, in organic solids, are essentially distinguished by the position of a proton within the crystal structure. While different chemical systems can appear in different forms, proton transfer has rarely been observed for multicomponent systems with identical stoichiometric composition. ${ }^{[3]}$

In this contribution, we present an extremely rare case of polymorphism between a metastable molecular (cocrystal) and ionic (salt) form of a two-component system based on nicotinamide and a dicarboxylic acid, induced by supramolecular tautomerism. In specific, we show the polymorphic transition from a metastable cocrystal to a salt, monitored using time-resolved powder X-ray diffraction (PXRD) and solid-state nuclear magnetic resonance spectroscopy. Both formerly unknown structures of were solved ab initio from PXRD data and further analyzed using spectroscopic methods, as well as density functional theory calculations.
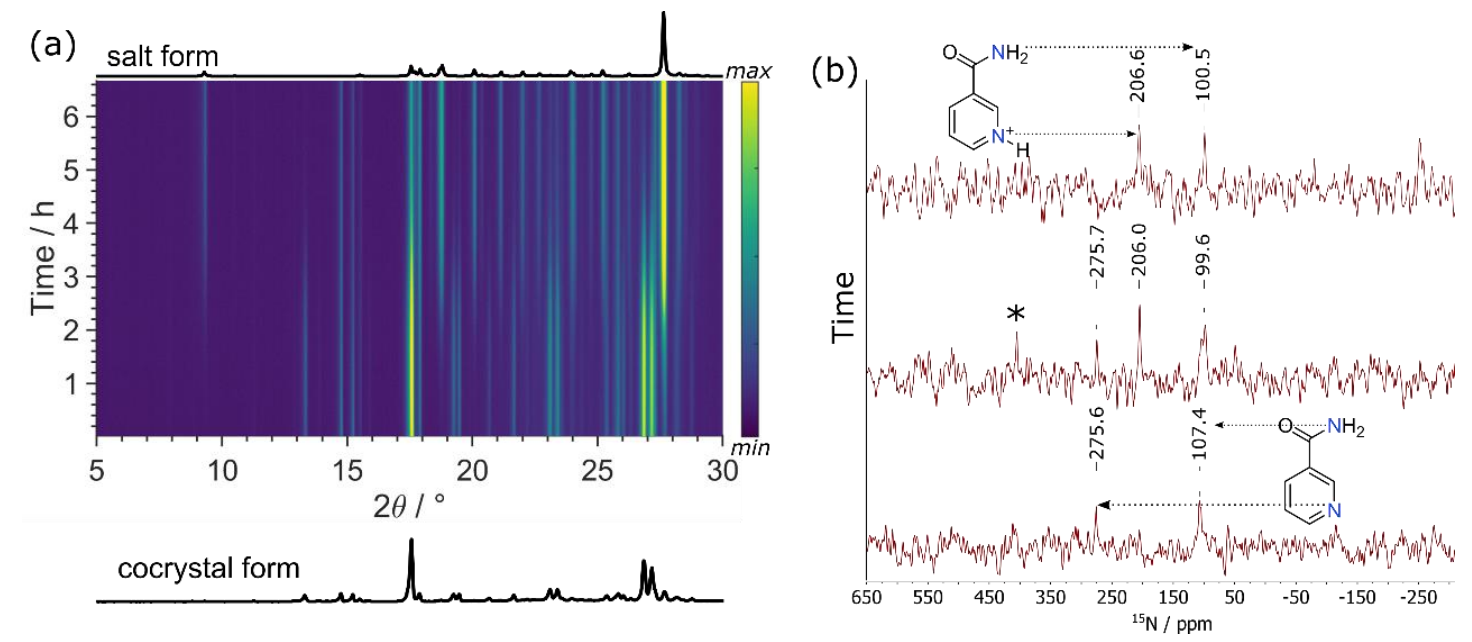

Figure 1: Monitoring the polymorphic transition from metastable cocrystal to salt using (a) PXRD and (b) ${ }^{15} \mathrm{~N}$ ssNMR spectroscopy.

[1] Almarsson, O. \& Zaworotko, M, J. (2004). Chem. Commun., 17, 1889-1896.

[2] Aitipamula, S. et al. (2014), Cryst. Growth Des., 12, 2147-2152

[3] Bernasconi, D., Bordignon, S., Rossi, F., Priola, E., Nervi, C., Gobetto, R., Voinovich, D., Hasa, D., Tuan Duong, N., Nishiyama, Y., Chierotti, M. R., (2020). Cryst. Growth Des., 20, 906-915.

Keywords: Cocrystals, polymorphism, supramolecular tautomerism, polymorphic transition, time-resolved monitoring. 